\title{
ANÁLISE DE PESTICIDAS EM AMOSTRAS AMBIENTAIS ORIUNDAS DA BARRAGEM DE BOA ESPERANÇA (PI/MA BRASIL): AVALIAÇÃO PRELIMINAR *
}

\author{
PAULO ROBERTO BRASIL DE OLIVEIRA MARQUES ** \\ GILVANDA SILVA NUNES ** \\ MARIA ELIANA RIBEIRO DE QUEIROZ *** \\ JOSÉ FÁBIO FRANÇA ORLANDA **** \\ HILDO SERAFIM DE SOUSA ***** \\ TERESA CRISTINA RODRIGUES DOS SANTOS
}

\begin{abstract}
A Barragem de Boa Esperança constitui importante potencial hídrico, social e econômico para a região Nordeste do Brasil, necessitando de monitoramento constante. Amostras de água e sedimentos foram coletadas e os princípios ativos Monocrotofós, Pirimicarb, Carbendazim, Triclorfon e Fenvalerato analisados por cromatografia a gás. Utilizou-se metodologia multirresíduo para extração dos pesticidas (FDA modificado) e cromatografia a gás (CG - DCE/DFC). A metodologia forneceu eficiência e linearidade, com recuperações entre 69 e $86 \%$ para amostras fortificadas. Fenvalerato e Triclorfon foram detectados em dois pontos de coleta de amostras. Não foram detectados nenhum dos pesticidas em amostras de sedimentos.
\end{abstract}

PALAVRAS-CHAVE: ÁGUA-CONTAMINAÇÃO; AGROTÓXICOS; SEDIMENTOS; CROMATOGRAFIA A GÁS.

\section{INTRODUÇÃO}

O uso do potencial hídrico de maneira adequada tem melhorado a qualidade de vida do ser humano. A alteração de regimes de escoamento para controle de vazões tem permitido consideráveis avanços na geração

* Extraído de parte da dissertação apresentada pelo primeiro autor à Universidade Federal do Maranhão, como requisito para obtenção do título de Mestre em Química Analítica.

** Professores, Departamento de Tecnologia Química, Núcleo de Análises de Resíduos de Pesticidas (NARP), Universidade Federal do Maranhão (UFMA), São Luís, MA.

*** Professora, Departamento de Química, Universidade Federal de Viçosa (UFV), Viçosa, MG.

**** Pós-graduando em Química, UFV, Viçosa, MG.

***** Aluno, Iniciação Científica, Curso de Química Industrial, UFMA, São Luís, MA.

****** Professora, Departamento de Tecnologia Química, NARP, UFMA, São Luís, MA. 
de energia elétrica, na pecuária, na aqüicultura e, especialmente, na agricultura. Porém, nos extensos cultivos agrícolas situados às margens dos compartimentos aquáticos são utilizados pesticidas para o controle de pragas danosas (1). Esses Agroquímicos têm sido estudados e considerados, em sua maioria, como potencialmente tóxicos ao homem, aos mamíferos, aos invertebrados e ao meio ambiente. Podem alcançar os ambientes aquáticos, prejudicar a biota, interferir na cadeia alimentar e, conseqüentemente prejudicar a saúde humana (2-4). Os pesticidas modernos, em particular os organofosforados e carbamatos, não apresentam o mesmo comportamento que os organoclorados, que se acumulam em gorduras. São primariamente transformados, em sistemas aquáticos, tendo baixo potencial de bioacumulação (5-7). Rios, lagos, barragens e mananciais podem ser contaminados por pesticidas, que seguem distintas rotas de contaminação. Tais rotas dependem das propriedades físico-químicas dos pesticidas bem como das propriedades do ambiente em estudo (8). A degradação dessas classes de pesticidas pode resultar em metabólitos muito mais prejudiciais que o próprio pesticida original (9-10). Assim sendo, técnicas sensíveis têm sido estudadas no sentido de monitorar a dinâmica dos pesticidas no ambiente. A cromatografia, com uso de distintos detectores e metodologias como as multirresiduais, vem sendo destacada como uma das ferramentas analíticas mais empregadas nesse tipo de estudo (11-16). Por conseguinte, estudos ambientais sobre impactos de pesticidas no Brasil têm sido apresentados na literatura (17-30).

Neste trabalho pretendeu-se classificar os pesticidas aplicados na região da Barragem de Boa Esperança e analisar amostras de água e sedimentos mediante metodologia de extração multirresidual e cromatografia a gás.

\section{MATERIAL E MÉTODOS}

\section{1 ÁREA DO ESTUDO}

O rio Parnaíba, com 1.480 km de comprimento, é a linha divisória entre os estados do Maranhão e Piauí (região Nordeste do Brasil), sendo sua bacia hidrográfica a quarta maior em área do país (31). A Barragem de Boa Esperança, formada pelo represamento do trecho médio do rio Parnaíba, também é alimentada pelo rio Balsas (MA) que deságua nas proximidades das cidades de Uruçuí (PI) e Benedito Leite (MA). A barragem encontra-se geograficamente situada entre as coordenadas 06ㄴㄷ' S e 434' W, com volume estimado em $5.085 \times 10^{-6} \mathrm{~m}^{3}$ de água e 
cerca de $100 \mathrm{~km}$ de extensão máxima (32). O clima da região é do tipo tropical, caracterizado por temperatura média anual superior a $18^{\circ} \mathrm{C}$. $\mathrm{O}$ regime de precipitação pluviométrica origina duas estações climatológicas bem definidas, ou seja, uma estação chuvosa (janeiro a junho) e outra de estiagem (julho a dezembro). O solo da região apresenta elevada profundidade e acentuada drenagem, com baixa fertilidade, alta acidez, altas taxas de infiltração e permeabilidade, além de baixa capacidade de retenção de umidade. A área, com precipitação média anual em torno de $1100 \mathrm{~mm}$, apresenta topografia com declive geralmente abaixo de $4 \%$ (33).

Estudos ambientais têm sido efetuados na região pelo Laboratório de Hidrobiologia (LABOHIDRO) e pelo Núcleo de Análises de Resíduos de Pesticidas (NARP), ambos da Universidade Federal do Maranhão, juntamente com a Companhia Hidroelétrica do São Francisco (CHESF) visando gerar dados mais detalhados pata monitoramento e gerenciamento ambiental.

\subsection{AMOSTRAGEM}

A amostragem foi efetuada em 6 pontos significativos da Barragem de Boa Esperança, nos períodos chuvoso (março) e de estiagem (setembro) do ano de 2000. Os pontos foram pré-determinados a partir de trabalhos anteriores de coleta e amostragem efetuados pelo LABOHIDRO (Tabela 1). As amostras de água foram coletadas em 6 pontos para superfície (A) e 5 pontos para fundo (B), com o auxílio de garrafa de Van Don. As amostras de fundo variaram entre 6 a 25 metros de profundidade entre os pontos de coleta. Uma amostra da água de canal de irrigação de área de plantio, que alimenta a barragem, foi coletada como amostra teste.

\section{TABELA 1 - PONTOS DE COLETA NA ÁREA DA BARRAGEM DE BOA ESPERANÇA}

\begin{tabular}{|c|c|c|c|c|c|c|}
\hline \multicolumn{7}{|c|}{ PONTOS DE COLETA } \\
\hline Floriano (PI) & Jusante & $\begin{array}{c}\text { Nova } \\
\text { Guadalupe (PI) }\end{array}$ & $\begin{array}{c}\text { Nova } \\
\text { lorque (MA) }\end{array}$ & $\begin{array}{l}\text { Benedito } \\
\text { Leite (MA) }\end{array}$ & Uru u (PI) & $\begin{array}{c}\text { Canal de } \\
\text { irriga }{ }^{a} \circ(\mathrm{PI})\end{array}$ \\
\hline
\end{tabular}

COORDENADAS (GPS)

$64555 \mathrm{~S} \quad 64528 \mathrm{~S} \quad 64540 \mathrm{~S} \quad 64555 \mathrm{~S} \quad 71402 \mathrm{~S} \quad 71420 \mathrm{~S}$

43" 0122 W 43" 3314 w 43 " 3440 W 44" 226 W 44" 3422 W 44 " 3408 W 
As amostras de água da primeira coleta apresentaram-se visualmente muito sujas, barrentas e com sólidos suspensos, enquanto que as da segunda, mostraram-se limpas com ótima transparência. Imediatamente após a coleta as amostras de água foram filtradas em filtro de nitrato celulose $0,45 \mathrm{~mm}$, preservadas com algumas gotas de ácido nítrico concentrado e acondicionadas em frascos de vidro âmbar $(1 \mathrm{~L})$ em refrigerador a - $4^{\circ} \mathrm{C}$. Esse tipo de água foi classificada como água de consumo direto, sem pré-tratamento (34).

As amostras de sedimento, coletadas com o auxílio de draga manual em 5 dos 6 pontos de coleta, variaram para cada estação. Apresentaram-se de arenosas a extremamente argilosas, sendo que a maioria continha elevada incidência de matéria orgânica. Os sedimentos foram acondicionados in natura em sacos de polietileno, sob refrigeração a - 4으. As amostras de água e de sedimento foram transportadas para o laboratório em refrigerador, sendo acondicionadas no mesmo sistema de refrigeração, $\mathrm{a}-4 \stackrel{\circ}{\circ}$.

A primeira coleta ocorreu sob condição chuvosa, com fraca incidência de luz solar e temperatura ambiente variando entre 29 e $30 \circ \mathrm{C}$. A segunda foi efetuada em período de alta incidência de luz solar com elevadas temperaturas ambientes (acima de $32^{\circ} \mathrm{C}$ ).

Efetuou-se junto ao setor agronômico responsável pela área, levantamento de informações acerca dos cultivos agrícolas, pragas combatidas e pesticidas utilizados. A partir destes dados, efetuou-se a descrição dos pesticidas em estudo segundo suas classes e toxicologia (Tabela 2).

\subsection{MATERIAL}

Foram utilizados os solventes acetona, acetonitrila, acetato de etila, metanol, hexano, diclorometano e sulfato de sódio grau HPLC e florisil®. Empregaram-se padrões dos pesticidas Monocrotofós, Pirimicarb, Carbendazim, Triclorfon e Fenvalerato da marca Dr. Ehrenstofer(Alemanha) com pureza superior a 95\%. Foi utilizada água deionizada obtida de sistema Milli-Q (Millipore, USA).

\subsection{PREPARO DOS EXTRATOS}

Utilizou-se o método de extração multirresidual proposto pela FDA, com algumas modificações (36) (Figura 1). Resumidamente, após extração com acetona, as amostras foram particionadas (PLL), purificadas em coluna de partição com Florisil@, parcialmente desativado, concentradas em rotaevaporador e os resíduos finais dissolvidos em acetato de etila. 
Essa solução foi acondicionada em frascos do tipo vials, recobertos por parafilm e guardados, em laboratório, sob refrigeração a $-4^{\circ} \mathrm{C}$. Os frascos foram acondicionados, em grupos, em depósitos de plásticos, que por sua vez, foram colocados em bolsas térmicas. As bolsas foram enviadas para o Laboratório de Química Ambiental da Universidade Federal de Viçosa, no qual foram efetuadas as determinações por cromatografia a gás (CG). As injeções cromatográficas foram otimizadas, inicialmente a partir de injeções em branco, seguido de injeções de padrões.

\section{TABELA 2 - PESTICIDAS MAIS APLICADOS NAS ÁREAS DE BARRAGEM DE BOA ESPERANÇA}

\begin{tabular}{|c|c|c|c|c|c|c|}
\hline $\begin{array}{l}\text { NOME } \\
\text { COMERCIAL }\end{array}$ & $\begin{array}{l}\text { PRINC"PIO } \\
\text { ATIVO }\end{array}$ & CULTURA & PRAGA & CLASSE & \multicolumn{2}{|c|}{$\begin{array}{c}\text { GRUPO } \\
\text { TOXICOLOGIA* }\end{array}$} \\
\hline Nuvacron & Monocrotof s & 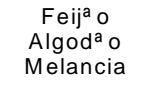 & $\begin{array}{c}\text { Pulga o } \\
\text { Cigarra } \\
\text { Vaquinha }\end{array}$ & $\begin{array}{l}\text { Inseticida } \\
\text { Acaricida } \\
\text { SistCfnico }\end{array}$ & $\begin{array}{l}\text { Organo- } \\
\text { fosforado }\end{array}$ & 1 \\
\hline Pi-rimor & Pirimicarb & $\begin{array}{l}\text { Feij }{ }^{\mathrm{a}} \text { o } \\
\text { Tomate }\end{array}$ & Pulg ${ }^{\mathrm{a}} 0$ & $\begin{array}{l}\text { Inseticida } \\
\text { Aficida }\end{array}$ & Carbamato & II \\
\hline Dipterex & Triclorfon & $\begin{array}{c}\text { Arroz } \\
\text { Ab bora } \\
\text { Tomate } \\
\text { Feij- } 0\end{array}$ & $\begin{array}{c}\text { Lagarta } \\
\text { Vaquinha } \\
\text { Mosca } \\
\text { Branca }\end{array}$ & $\begin{array}{l}\text { Inseticida } \\
\text { Contato }\end{array}$ & $\begin{array}{l}\text { Organo- } \\
\text { fosforado }\end{array}$ & II,III \\
\hline Bendazol & Carbendazim & Trigo & Giberela & $\begin{array}{l}\text { Fungicida } \\
\text { SistCfrn ico }\end{array}$ & Benzimidazol & III \\
\hline Fenvalerato & Fenvalerato & $\begin{array}{c}\text { Caf } \varnothing \\
\text { Algod } \\
\text { Soja }\end{array}$ & $\begin{array}{c}\text { Lagarta } \\
\text { Percevejo } \\
\text { Bicho } \\
\text { Mineiro }\end{array}$ & $\begin{array}{l}\text { Inseticida } \\
\text { fitosanit/\&io }\end{array}$ & $\begin{array}{l}\text { Piretr ide } \\
\text { SintØtico }\end{array}$ & 1 \\
\hline
\end{tabular}

* Toxicidade segundo a Organização Mundial de Saúde (35): I = extremamente tóxico; II = moderadamente tóxico e III = levemente tóxico.

\subsubsection{Condições cromatográficas otimizadas para os pesticidas organofosforados, carbamatos e benzimidazóis}

Foi utilizado cromatógrafo da marca Shimadzu, modelo GC 17A, com detector fotométrico de chama (DFC) a $225-250^{\circ} \mathrm{C}$. A temperatura de trabalho do injetor foi de $250^{\circ} \mathrm{C}$, empregou-se coluna capilar DB-17 (30 m x $0,25 \mathrm{~mm}$ d.i. e $1,5 \mu \mathrm{m}$ de espessura de filme), com temperatura isotérmica de $200^{\circ} \mathrm{C}$. O volume de injeção foi de $1 \mu \mathrm{L}$, sendo usado Hélio (He) ultrapuro como gás de arraste, em fluxo de $10 \mathrm{~mL} / \mathrm{min}$ e razão de split de 1:1. 
FIGURA 1 - ESQUEMA PARA PREPARO DOS EXTRATOS PARA ANÁLISE CROMATOGRÁFICA

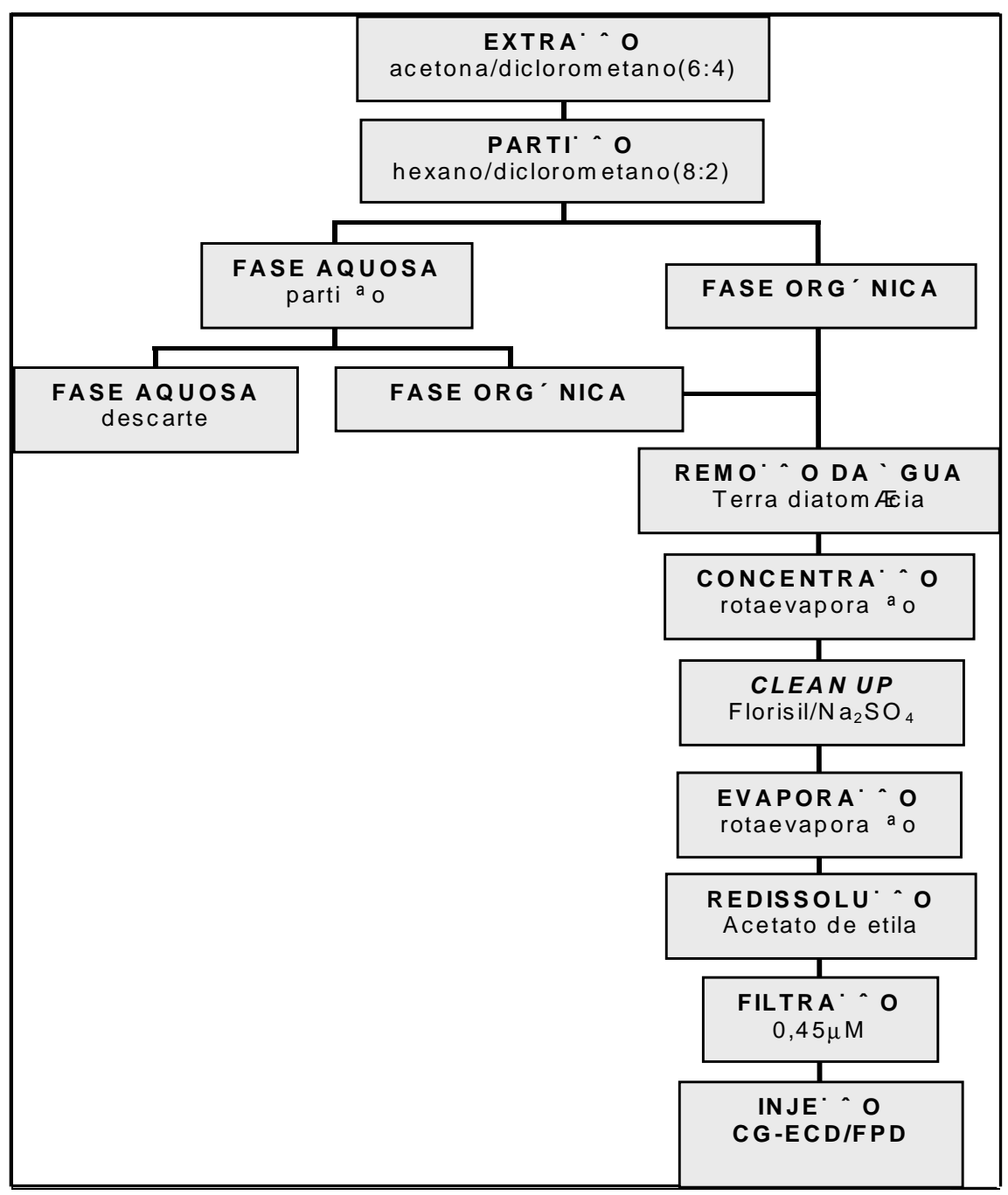

\subsubsection{Condições cromatográficas otimizadas para o piretróide sintético Fenvalerato}

Para o piretróide Fenvalerato empregou-se o mesmo cromatógrafo, com detector de captura de elétrons (DCE) a $350^{\circ} \mathrm{C}$ e temperatura do 
injetor de $220-250{ }^{\circ} \mathrm{C}$. Utilizou-se coluna mega bore DB-1 (30 m x $0,53 \mathrm{~mm}$ d.i. e 1,5 $\mu \mathrm{m}$ de espessura de filme), em temperatura de eluição isotérmica de $200{ }^{\circ} \mathrm{C}$. O volume de injeção foi de $1 \mu \mathrm{mL}$. Utilizou-se a mistura $\mathrm{N}_{2} /$ metano (95:5) como gás de arraste, com fluxo de gás de $15 \mathrm{~mL} / \mathrm{min}$. A razão split foi de 1:1.

\section{RESULTADOS E DISCUSSÃO}

A Figura 2 apresenta o cromatograma obtido da mistura padrão de quatro dos pesticidas estudados. A Figura 3 refere-se a solução de padrão do pesticida Fenvalerato.

\section{FIGURA 2 - CROMATOGRAMA (CG-DFC) DA MISTURA DE PADRÕES (1 mg.L-1), PREPARADOS POR DISSOLUÇÃO DE SOLUÇÕES ESTOQUE (EM METANOL) EM ACETATO DE ETILA}

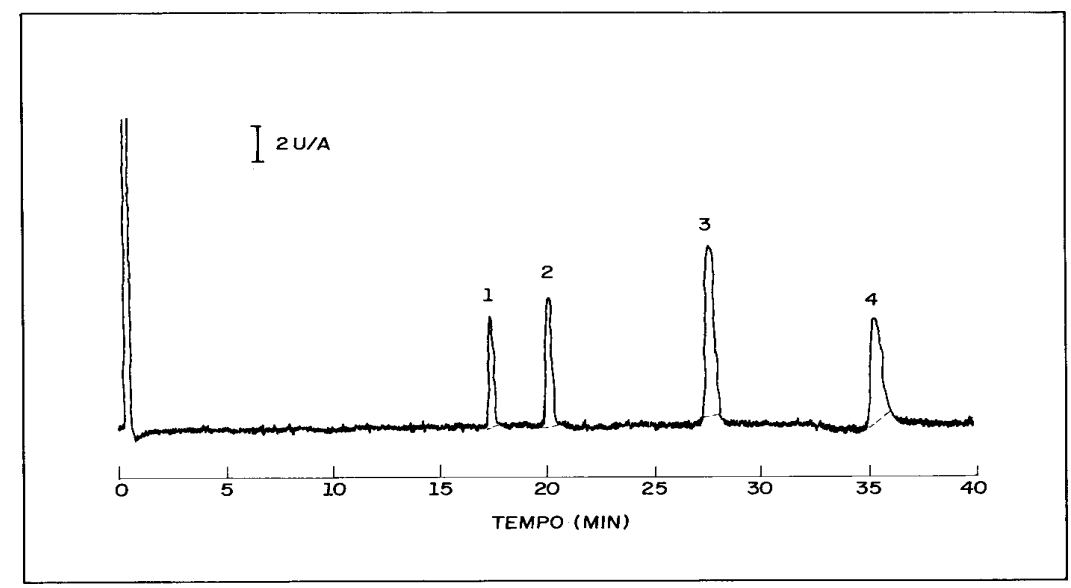

1 = Monocrotofós; 2 = Triclorfon; 3 = Pirimicarb; 4 = Carbendazim .

Os cálculos de área foram realizados mediante injeção em duplicata de cada extrato, sendo a concentração do princípio ativo determinada por meio de curvas de calibração (Figura 4). Verificou-se, inicialmente, a linearidade da curva de calibração, porém não foram injetados os padrões mais concentrados, uma vez que as concentrações presentes nas amostras encontravam-se em níveis muito baixos. 
FIGURA 3 - CROMATOGRAMA REFERENTE AO PADRÃO

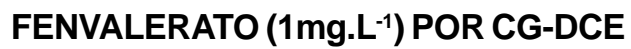

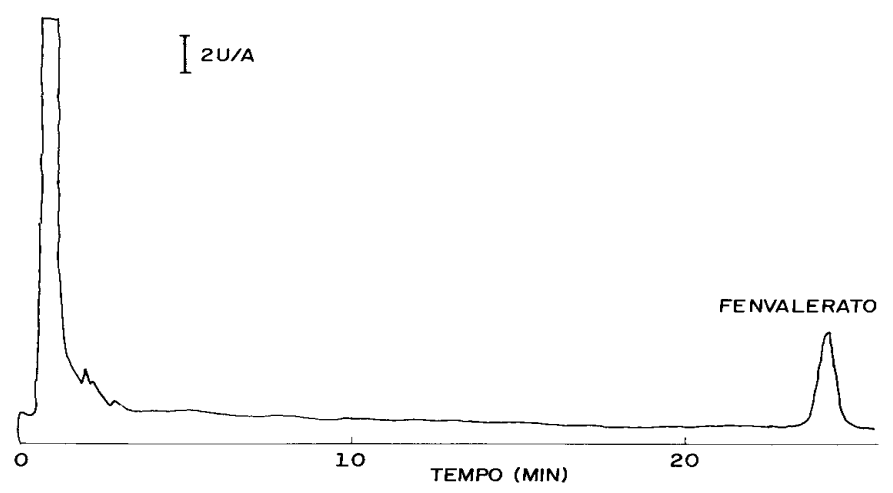

FIGURA 4 - CURVA DE CALIBRAÇÃO PARA O PESTICIDA METAMIDOFÓS

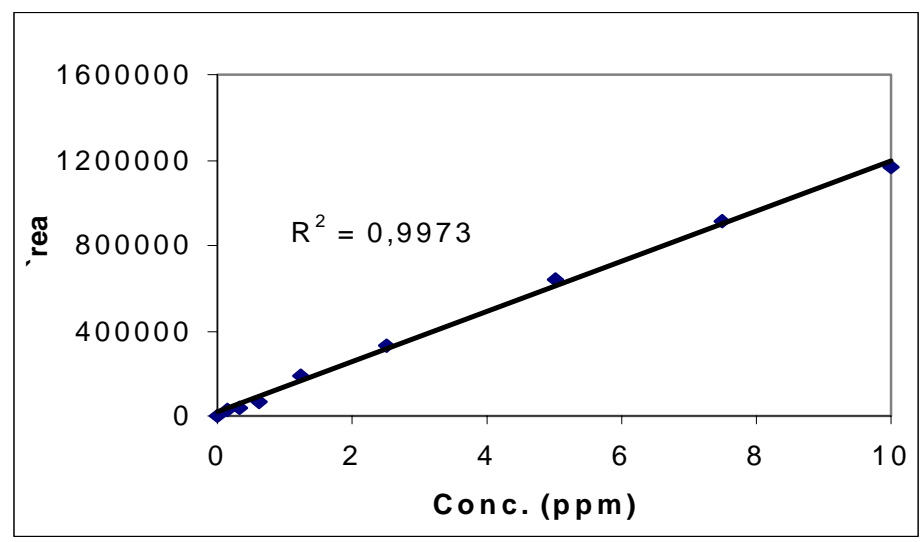


As condições cromatográficas foram ajustadas, inicialmente, a partir de várias injeções do branco, soluções de padrões e amostras, de modo que os compostos de interesse fossem eluídos após os interferentes (substâncias co-extraídas que não foram eliminadas durante a etapa de purificação dos extratos), isto é, em tempos de retenção (tR) maiores.

Preparou-se amostra com a matriz isenta de ativos (branco) nas mesmas condições de extração. A reprodutibilidade dos tempos de retenção mostrou-se excelente (Tabela 3). A faixa de trabalho para os compostos foi adequada para a análise mediante a metodologia proposta uma vez que a concentração final dos extratos foi ajustada para que 0 detector pudesse "enxergar" os picos de interesse. No presente estudo o extrato final foi concentrado 100 vezes para atingir a sensibilidade desejada.

\section{TABELA 3 - TEMPOS DE RETENÇÃO (TR) E LIMITES DE DETECÇÃO DOS PESTICIDAS ANALISADOS PELO SISTEMA CG/ DFC}

\begin{tabular}{|c|c|c|c|}
\hline PESTICIDA & $T_{R}$ & $\begin{array}{c}\text { CONCENTRA } \\
(\mathrm{ng})\end{array}$ & $\begin{array}{l}\text { LIMITE DE DETEC`^ O } \\
(\mathrm{ng})^{\star}\end{array}$ \\
\hline Monocrotof s & $17,9-18,1$ & $0,5-2,3$ & 0,3 \\
\hline Triclorfon & $20,1-20,3$ & $0,42-3,5$ & 0,36 \\
\hline Pirimicarb & $27,5-27,8$ & $0,22-2,8$ & 0,15 \\
\hline Carbendazim & $35,4-35,7$ & $0,25-3,3$ & 0,18 \\
\hline
\end{tabular}

*Médias de 3 repetições.

Os tempos de retenção e limites de detecção do Fenvalerato, analisado pelo sistema CG/DCE oscilaram entre 23,9 e $24,1 \mathrm{~min}$, com limite de detecção de $0,05 \mathrm{ng}$, em médias de 3 repetições.

Duas amostras, nas quais não foram previamente detectados os princípios ativos estudados, foram escolhidas como testemunhas e fortificadas com $1 \mathrm{mg} / \mathrm{L}$ de padrões (Figura 5). Os tempos de retenção dos compostos em todos os extratos fortificados, bem como dos nãofortificados, apresentaram os tempos de retenção dos padrões.

A eficiência do método foi calculada com base nas médias das recuperações de todos os compostos em amostras fortificadas, que variou entre 69 a 86\% (Tabela 4). As médias mostraram-se adequadas para os tipos de resíduos estudados em amostras ambientais, considerando-se os efeitos de matriz. 
FIGURA 5 - CROMATOGRAMA (CG-DFC) DE UMA AMOSTRATESTEMUNHA (NOVA GUADALUPE- PI, AMOSTRA DE SUPERFÍCIE), FORTIFICADA COM 1 mg.L-1 DA MISTURA DE PADRÕES

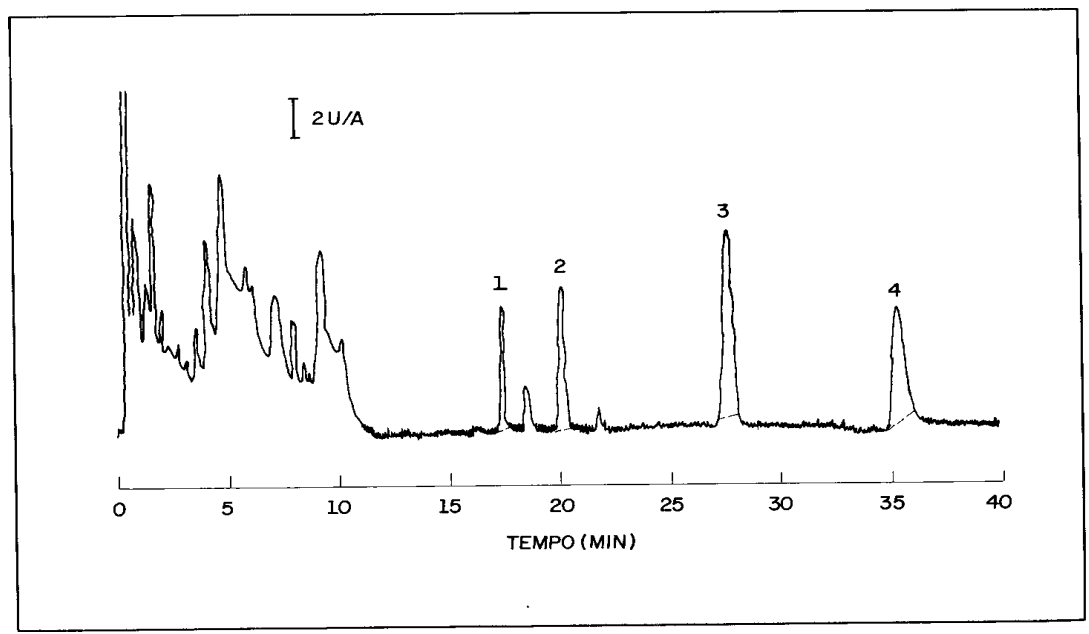

1 - Monocrotofós; 2 - Triclorfon; 3 - Pirimicarb; 4 - Carbendazim.

TABELA 4 - RECUPERAÇÃO DO TUEOR DE ATIVO DOS PESTICIDAS NAS AMOSTRAS DE ÁGUA

\begin{tabular}{|c|c|c|c|}
\hline PESTICIDA & $\begin{array}{c}\text { CONCENTRA }^{\text {DE FORTIFICA }} \cdots \\
\text { DE } \\
\left(\mathrm{mg}^{-1} \mathrm{~L}^{-1}\right)\end{array}$ & $\begin{array}{l}\text { CONCENTRA ^O } \\
\text { DETECTADA } \\
\left(m g \cdot \mathrm{L}^{-1}\right)\end{array}$ & $\begin{array}{c}\text { RECUPERA ^ } 0 \\
(\%)\end{array}$ \\
\hline Monocrotof s & 1 & & \\
\hline Pirimicarb & 1 & 0,722 & 72,2 \\
\hline Triclorfon & 1 & 0,690 & 69,0 \\
\hline Carbendazim & 1 & 0,815 & 81,5 \\
\hline Fenvalerato & 1 & 0,754 & 75,4 \\
\hline Fenvalerato & 1 & 0,862 & 86,2 \\
\hline
\end{tabular}

*Médias de 2 repetições.

Dos princípios ativos estudados apenas o Triclorfon e o Fenvalerato foram detectados, tanto em amostras de água de superfície quanto de fundo nos pontos de coleta Nova lorque (MA) e no canal de irrigação(PI), mas somente no período chuvoso. Os demais pesticidas apresentaramse abaixo do limite de detecção do método (Tabela 5). 
TABELA 5 - TEORES DE PESTICIDAS DETECTADOS EM AMOSTRAS DE ÁGUA, NA BARRAGEM DE BOA ESPERANÇA

\begin{tabular}{|c|c|c|}
\hline Pesticidas & {$\left[\mu \mathrm{g} \cdot \mathrm{L}^{-1}\right]^{*}$} & Ponto de coleta \\
\hline \multirow[t]{2}{*}{ Tricorfon } & 34,5 & $\underset{\text { Nova lorque (MA) }}{\text { Nove }}$ \\
\hline & 15,5 & $\underset{\mathbf{B}}{\text { Nova lorque }}(\mathrm{MA})$ \\
\hline \multirow[t]{3}{*}{ Fenvalerato } & 11,9 & $\underset{\mathbf{A}}{\text { Nova lorque }}(\mathrm{MA})$ \\
\hline & 5,7 & $\underset{\mathbf{B}}{\text { Nova lorque }}$ \\
\hline & 7,0 & Canal de Irriga ${ }^{a}$ \\
\hline
\end{tabular}

"Média de 2 determinações. A - amostras de superfície; B - amostras de fundo.

A Figura 6 mostra o cromatograma de uma das amostras de água, a qual apresentou resíduo do pesticida Triclorfon. Pode-se observar que o tempo de retenção(TR) do composto está distanciado dos demais, indicando que os co-extrativos que poderiam interferir na análise foram eluídos antes do pesticida detectado.

FIGURA 6 - CROMATOGRAMA (CG-DFC) DE TRICLORFON, DETECTADO EM AMOSTRA DE ÁGUA DE FUNDO, PONTO DE COLETA - NOVA IORQUE

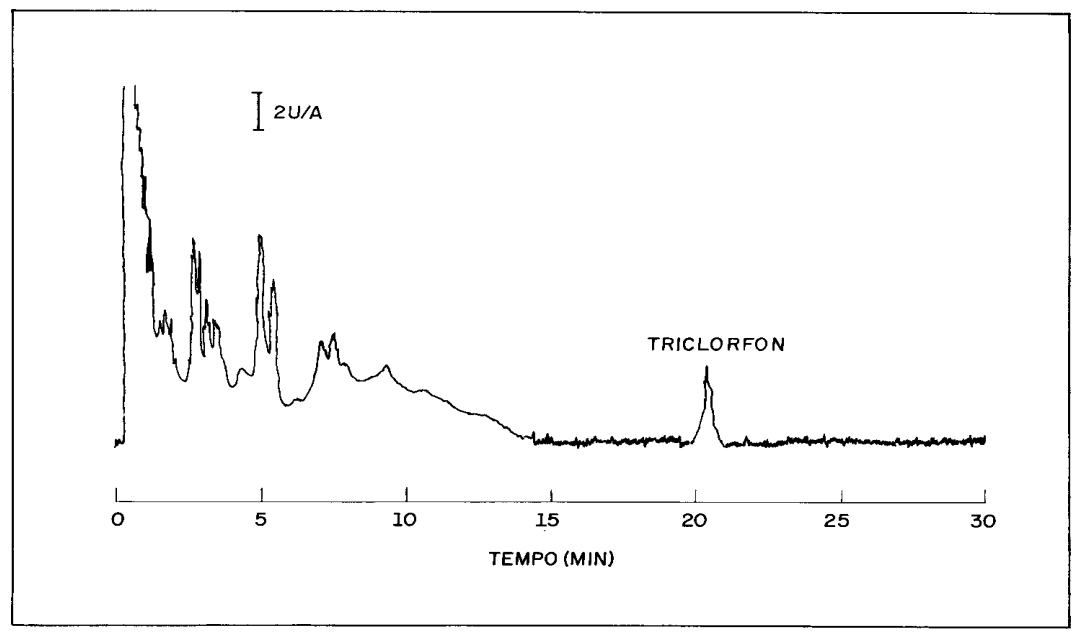


As propriedades físico-químicas dos pesticidas analisados neste trabalho revelam que todos podem apresentar rotas de contaminação para o meio aquático (Tabela 6). O Fenvalerato e o Triclorfon podem ter seguido essas rotas, visto que foram aplicados cerca de 15 dias antes do período de coleta, durante a estação chuvosa, em localidades distantes do ponto detectado.

\section{TABELA 6 - PRINCIPAIS PROPRIEDADES FÍSICO-QUÍMICAS DOS PRINCÍPIOS ATIVOS ESTUDADOS}

\begin{tabular}{|c|c|c|c|c|}
\hline \multirow{2}{*}{ Pesticida } & Solubilidade em & \multirow{2}{*}{$\begin{array}{l}\text { Press }^{\mathrm{a}} \text { o de vapor } \\
(\mathrm{mPa} 20 " \mathrm{C})\end{array}$} & \multirow{2}{*}{$\begin{array}{c}\text { Parti a o } \\
\text { Afgua octanol }\end{array}$} & \multirow{2}{*}{$\begin{array}{c}\text { Constante de } \\
\text { ioniza a o frido- } \\
\text { base }\left(P_{k a}\right)\end{array}$} \\
\hline & gua (mg/L 20 "C) & & & \\
\hline Carbendazim & 8 & 0.09 & 32 & 4.2 \\
\hline Fenvalerato & $<0.01$ & 0.192 & 5.01 & - \\
\hline Monocrotof $\mathrm{s}$ & $100 \%$ & 29 & -0.22 & - \\
\hline Pirimicarb & $3 \times 10^{3}$ & 0.97 & 1.7 & - \\
\hline Triclorfon & $120 \times 10^{3}$ & 0.21 & 0.43 & - \\
\hline
\end{tabular}

O piretróide sintético Fenvalerato é estável em meio ácido, que é a característica do tipo de solo da região. Tal solo apresenta, ainda, baixa capacidade de retenção de umidade, altas taxas de infiltração com acentuada drenagem, e baixo teor de matéria orgânica. Essas características podem ter levado o pesticida a atingir o meio aquático. As condições de chuvas da estação, como altos índices pluviométricos também devem ter facilitado a lixiviação do composto para o compartimento aquático. As condições de clima nublado podem ter ocasionado maior persistência do princípio ativo no meio aquático, nesse período, devido ao baixo índice de fotólise. O ponto de coleta 4 apresentou águas sem perturbações, o que pode ter facilitado o transporte do pesticida na coluna d'água.

A amostra-teste de número 7 , coletada no canal de irrigação alimentado pelas águas da Barragem, foi provavelmente contaminada por rotas aéreas de exposição, no que diz respeito à aplicação do produto nas plantações ou por excesso de agroquímico aplicado nas culturas. $O$ próprio canal serviria como veículo de contaminação, visto que o contaminante pode retornar à Barragem por lixiviação ou carreamento. $O$ terreno nas proximidades apresenta pequeno declive de $4^{\circ}$, propiciando contaminação das águas da Barragem.

A alta mobilidade do Tricorfon no solo, aliada às características do solo da região podem ter favorecido o transporte do pesticida para a 
água. O baixo índice de fotólise e pressão de vapor do produto devem ter contribuído, significativamente, para a persistência do pesticida no ambiente aquático no período chuvoso de coleta.

O período de estiagem apresentou temperaturas muito altas, quando comparadas ao período chuvoso. A irradiação solar foi intensa em todo o período da coleta, bem como nas semanas que antecederam a mesma. Esses fatores, somados à ausência de chuvas devem ter dificultado o transporte dos pesticidas aplicados nas culturas agrícolas do solo para a água. É possível que tenha ocorrido em grande extensão a fotodegradação dos compostos, visto que não foram detectados resíduos de pesticidas na segunda coleta.

Não foram detectados princípios ativos em nenhuma das amostras de sedimento. O ponto de coleta 4, no qual foram detectados resíduos de pesticidas tanto em amostras de água de superfície quanto de fundo não apresentou resíduo de nenhum composto estudado em sedimentos. Provavelmente o alto teor de argila e matéria orgânica podem ter acelerado a degradação dos mesmos, caso tenham alcançado este compartimento. Os demais sedimentos apresentaram-se arenosos, o que carateriza pouca capacidade adsortiva frente aos pesticidas. Desta forma, não devem ter sido fixados, contribuindo para a biodisponibilidade do inseticida na coluna d'água.

\section{CONCLUSÃO}

A reprodutibilidade dos tempos de retenção, a eficiência do método analítico empregado nesta etapa de trabalho e a linearidade para os compostos estudados foram adequadas, embora envolvam poucos princípios ativos. A coleta realizada na estação chuvosa parece ter favorecido a movimentação dos compostos detectados, através dos corpos d'água, uma vez que foram encontrados pesticidas em áreas bem distantes das zonas em que ocorreram as aplicações. Até mesmo as fortes chuvas devem ter ocasionado a lixiviação dos agroquímicos. Os princípios ativos estudados na estiagem podem ter sofrido influência da falta de chuvas.

Triclorfon e Fenvalerato foram detectados em faixas de concentração variando de 7 a $35 \mu \mathrm{g} \cdot \mathrm{L}^{-1}$, tanto em amostras de água de superfície, quanto de fundo, na primeira amostragem. Deve-se ressaltar que os teores detectados encontram-se acima dos limites permitidos por lei internacional $\left(0,1 \mu \mathrm{g} \cdot \mathrm{L}^{-1}\right.$ para pesticidas individuais e $0,5 \mu \mathrm{g} \cdot \mathrm{L}^{-1}$ para pesticidas totais) para esse tipo de água, evidenciando o uso indiscriminado e descontrolado de pesticidas na região. Não foram detectados resíduos de nenhum dos compostos estudados nas amostras provenientes da segunda coleta, nem nas amostras de sedimentos. 
Dada a importância da Barragem de Boa Esperança para a região Nordeste faz-se necessário o monitoramento constante e a avaliação dos riscos de contaminação, de forma a minimizá-los nesse importante ecossistema aquático. Além disso, a confirmação dos pesticidas encontrados em amostras de água, mediante técnicas cromatográficas empregando detecção por espectrometria de massas (CG/MS e/ou HPLC/ MS) podem complementar as informações analíticas, inclusive com o estudo dos produtos de degradação presentes e suas rotas de contaminação.

\section{Abstract}

ANALYSIS OF PESTICIDES IN ENVIRONMENTAL SAMPLES (WATER AND SEDIMENTS) OF THE BOA ESPERANÇA BARRAGE: BRAZIL - PI/MA: PRELIMINARY AVALIATION

Boa Esperança barrage is a very important social and economical region for northeast of Brazil, and it needs continuous enviromental monitoring. Water and sediments samples were collected in order to determine the most commonly used pesticides Monocrotophos, Pirimicarb, Carbendazim, Trichlorfon and Fenvalerate. It was employed the modified FDA multiresidue extraction method, followed by gas chromatography analysis (GC - FID/ FPD). This method provided good efficiency and adequate linearity, with recovery ranging from 69 to $86 \%$ for fortified samples. Fenvalerate and Trichlorfon were detected in two points of sampling. The selected pesticides have not been detected in sediments.

KEYWORDS: WATER-CONTAMINATION; PESTICIDES; SEDIMENTS; GAS CHROMATOGRAPHY.

\section{REFERÊNCIAS}

1 MACEDO, J. A. B. Águas e águas. São Paulo: Varela, 2001. 505 p.

2 O'NEIL, P. Environmental chemistry. $2^{\text {nd }}$ ed. London: Chapman \& Hall, 1993. $233 p$.

3 NUNES, G. S; RIBEIRO, M. L. Pesticidas: uso, legislação e controle. Pesticidas: R. Ecotox. e Meio Ambiente, Curitiba, v. 9, p. 31-44, jan./dez. 1999.

4 BRASIL usa e abusa dos agrotóxicos. Folha de São Paulo, São Paulo, 3 mar. 1998. Agrofolha, Caderno 5.

5 BOTKLIN, D. B.; KELLER, E. A. Environmental science: earth as a living planet. $3^{\text {rd }}$ ed. New York: Wiley, 2000. 
6 SHREVE, R. N.; BRINK JR., J. A. Indústria de processos químicos. 4.ed. Rio de Janeiro: Guanabara dois, 1980.

7 KAMRIN, M. A. Pesticides profile: toxicity, environmental impact and fate. New York: Lewis, 1997. p. 675.

8 DORES, E.F.G.C.; LAMONICA-FREIRE, E.M. Contaminação de ambientes aquáticos por pesticidas: vias de contaminação e dinâmica dos pesticidas no ambiente aquático. Pesticidas: R. Ecotox. e Meio Ambiente, Curitiba, v. 9, p. 1-18, jan./dez. 1999.

9 BARCELÓ, D.; HENNION, M.C. Trace determination of pesticides and their degradation products in water. Amsterdan: Elsevier, 1997.

10 BARCELÓ, D. Environmental protection-agency and other methods for the determination of pesticides and other trasnformation products in water. J. Chromatogr. B, v. 643, p. 117-143, Jul. 1993.

11 LARTIGES, S.B.; GARRIGUES, P. Gas chromatographic analysis of organophosphorus and organonitrogen pesticides with different detectors. Analysis, v. 23, n. 8, p. 418-421, 1995.

12 HONG, J. S. et al. Simultameous analysis of 25 pesticides in crop using gas chromatography and their identifiction by gas chromatography-mass spectrometry. J. Chromatogr., v. 639, n. 2, p. 261-271, 1993.

13 SEIBER, J. N.; GLOTFELTY, D. E.; LUCAS, A. D. et al. A multiresidue method by high-performance liquid chromatographybased fractionation and gas-chromatographic determination of trace levels of pesticides in air and water. Arch. Environ. Com. Tox., v.19, n. 4, p. 583-592, Jul./Ago. 1990.

14 SALAU, J. S. et al. Application of solid-phase disk extration followed by gas and liquid chromatography for the simultaneous fungicides: captan, captafol, carbendazim, chlorothalonil, ethirimol, folpet, metalaxyl and vinclozolin in environmental waters. Anal. Chim. Acta, v. 293 , n. 2, p. 109-117, 1994.

15 BICCHI, C.; DAMATO, A.; BALBO, C. Multiresidues for the quantitative gas chromatographic determination of pesticides residues 
in sweet cherries. J. AOAC. Inter., v. 80, n. 6, p. 1281-1286, Nov./ Dez. 1997.

16 OKIHASHI, M. et al. Development of simultaneous analysis for organonitrogen and pyrethroid pesticides with GC MS. J. Food Hyg. Soc. Jap., v. 35, n. 3, p. 258-261, 1994.

17 PEVELING, R. et al. Environmental impact of the locust control agents fenitrothion, fenitrothion-esfenvalerate and triflumuron on terrestrial arthropods in Madagascar. Crop Protection, v.18, n.10, p.659-676, Dez.1996.

18 VANDER WERF, H. M. G., Assessing the impact of pesticides on the environment. Agr. Ecosyst. Environ., v.60, n. 2-3, p. 81-96, Dez.1996.

19 TAYAPUTCH, N., Present aspects and environmental impacts of pesticide use in Thailand. J. Pestic. Sci., v. 21, n. 1, p. 132-135, 1996.

20 RAMALHO, J. F. G. P., et al.Contaminação da microbacia de Caetés com metais pesados pelo uso de agroquímicos. Pesq. Agropec. Bras., v.35, n.7, Jul. 2000.

21 DORES, Eliana Freire Gaspar de Carvalho; DE-LAMONICA-FREIRE, Ermelinda Maria. Contaminação do ambiente aquático por pesticidas. Estudo de caso: águas usadas para consumo humano em Primavera do Leste, Mato Grosso: análise preliminar. Quím. Nova, v.24, n.1, p.27-36, fev. 2001.

22 FILIZOLA, Heloisa Ferreira; FERRACINI, Vera Lúcia; SANS, Luís Marcelo Aguiar et al. Monitoramento e avaliação do risco de contaminação por pesticidas em água superficial e subterrânea na região de Guaíra. Pesq. Agropec. Bras., v. 37, n.5, p.659-667, maio 2002.

23 NAKAGAWA, Lia Emi; ANDRÉA, Mara Mercedes de. Liberação de resíduos não-extraíveis ou ligados do herbicida atrazina em solo e sua absorção por plantas. Pesq. Agropec. Bras., , v.35, n.8, p.15171522, ago.2000.

24 NAKAGAWA, Lia Emi; ANDRÉA, Mara Mercedes de. Degradação 
e formação de resíduos não-extraíveis ou ligados do herbicida atrazina em solo. Pesq. Agropec. Bras., v.35, n.8, p.1509-1515, ago. 2000.

25 RAMALHO, Jair Felipe Garcia Pereira; AMARAL SOBRINHO, Nelson Moura Brasil do; VELLOSO, Ary Carlos Xavier. Contaminação da microbacia de Caetés com metais pesados pelo uso de agroquímicos. Pesq. Agropec. Bras., v.35, n.7, p.1289-1303, jul. 2000.

26 PRADO, Alexandre G. S., VIEIRA, Eny M.; REZENDE, M. Olímpia de $O$. Monitoring of the harmful concentrations of 2,4dichlorophenoxyacetic acid (2,4-D) in soils with and without organic matter. J. Braz. Chem. Soc., v.12, n.4, p.485-488, Aug. 2001.

27 ZUIN, Vânia Gomes; AIROLDI, Flavia Pereira da Silva; NASCIMENTO, Nádia Regina do et al. Determination of pentachlorophenol and hexachlorobenzene in natural waters affected by industrial chemical residues. J. Braz. Chem. Soc., v.10, n.1, p.25-30, Jan.1999.

28 PAUMGARTTEN, Francisco José Roma; DELGADO, Isabella Fernandes; OLIVEIRA, Elba Santos et al. Levels of organochlorine pesticides in the blood serum of agricultural workers from Rio de Janeiro State, Brazil. Cad. Saúde Pública, v.14, suppl.3, p.33-39, 1998.

29 BRÉGA, Salete Marcia; VASSILIEFF, Igor; ALMEIDA, Alaor et al. Clinical, cytogenetic and toxicological studies in rural workers exposed to pesticides in Botucatu, São Paulo, Brazil. Cad. Saúde Pública, v.14, suppl.3, p.117-123, 1998.

30 SILVA, Mendonça; GULNAR, Azevedo e. Measuring exposure to organochlorinated pesticides. Cad. Saúde Pública, v.14, suppl. 3, p.177-179, 1998.

31 MARANHÃO. Secretaria do Estado do Meio Ambiente e Turismo (SEMATUR). Diagnóstico dos principais problemas ambientais do estado do Maranhão. São Luís, 1991. p. 193.

32 CHESF. Companhia Hidroelétrica do São Francisco. Departamento de Meio Ambiente. Plano de manejo ambiental do reservatório da Boa Esperança. São Luís,1994. p. 28.

33 LEITE, A. C. Aspectos agro-industriais do Estado do Maranhão. 
São Luís: Secretaria da Agricultura do Estado do Maranhão/ Departamento de Pesquisa e Experimentação, 1976. p. 33.

34 BRASIL. Conselho Nacional do Meio Ambiente. Resolução CONAMA n.20, de 18 de junho de 1986. Diário Oficial [da] República Federativa do Brasil, Brasília, 30 jul. 1986. 21 p.

35 OMS. Organização Mundial da Saúde. Guías para la calidad del água potable. 2.ed. Genebra, 1995. v. 1 (Recomendaciones).

36 FDA. Food and Drug Administration. Pesticide analytical manual. Washington, 1999. v. 1.

\section{AGRADECIMENTOS}

Os autores agradecem à Companhia Hidroelétrica de São Francisco (CHESF) pela oportunidade de trabalho na região da barragem e apoio logístico oferecido durante as etapas de coleta. Agradecem também ao Laboratório de Hidrobiologia da Universidade Federal do Maranhão, ao Laboratório de Química da Universidade Federal de Viçosa, pelo apoio durante as etapas de análise cromatográfica e ao CNPq, pela concessão da bolsa de pesquisa. 